\title{
Cryogenic pellet launcher adapted for controlling of tokamak plasma edge instabilities
}

\author{
P. T. Lang, P. Cierpka, J. Harhausen, J. Neuhauser, \\ C. Wittmann, and ASDEX Upgrade Team \\ Max-Planck-Institut für Plasmaphysik, EURATOM Association, Boltzmannstrasse 2, 85748 \\ Garching, Germany \\ K. Gál, S. Kálvin, G. Kocsis, J. Sárközi, and T. Szepesi \\ KFKI-RMKI, EURATOM Association, P.O. Box 49, H-1525 Budapest-114, Hungary \\ C. Dorner and G. Kauke \\ Fachhochschule Regensburg, Fachbereich Maschinenbau, Galgenbergstrasse 30, 93053 \\ Regensburg, Germany
}

(Received 8 November 2006; accepted 30 December 2006; published online 27 February 2007)

\begin{abstract}
One of the main challenges posed recently on pellet launcher systems in fusion-oriented plasma physics is the control of the plasma edge region. Strong energy bursts ejected from the plasma due to edge localized modes (ELMs) can form a severe threat for in-vessel components but can be mitigated by sufficiently frequent triggering of the underlying instabilities using hydrogen isotope pellet injection. However, pellet injection systems developed mainly for the task of ELM control, keeping the unwanted pellet fueling minimized, are still missing. Here, we report on a novel system developed under the premise of its suitability for control and mitigation of plasma edge instabilities. The system is based on the blower gun principle and is capable of combining high repetition rates up to $143 \mathrm{~Hz}$ with low pellet velocities. Thus, the flexibility of the accessible injection geometry can be maximized and the pellet size kept low. As a result the new system allows for an enhancement in the tokamak operation as well as for more sophisticated experiments investigating the underlying physics of the plasma edge instabilities. This article reports on the design of the new system, its main operational characteristics as determined in extensive test bed runs, and also its first test at the tokamak experiment ASDEX Upgrade. () 2007 American Institute of Physics.
\end{abstract}

[DOI: $10.1063 / 1.2437116]$

\section{INTRODUCTION}

In recent decades injection of pellets made from cryogenic solid hydrogen isotopes (H: protium ${ }^{1} H$; D: deuterium ${ }^{2} H$; T: tritium ${ }^{3} H$ ) has been used in fusion-oriented plasma physics essentially for the purpose of particle refueling. ${ }^{1}$ Investigations on pellet-based refueling in hot tokamak plasmas reaching the high-confinement $(H$-mode) regime unveiled several phenomena showing strong impact on the fueling and hence plasma performance. ${ }^{2}$ Two of the most crucial are the curvature-induced drift of the high-pressure plasmoid forming around the ablating pellet ${ }^{3,4}$ and the pelletinduced release of edge localized modes (ELMS). ${ }^{5}$ The first one causes a fast particle and energy transport connected to the pellet ablation toward the plasma low-field side (LFS), making pellet launch from the magnetic high-field side (HFS) much more efficient for fueling. ${ }^{6}$ The second one causes additional prompt particle and energy losses from the plasma edge region, penalizing shallow pellet particle deposition profiles as generated by LFS injection, making this injection scheme even more unfavorable. Hence, conventional pellet launch injecting from the torus LFS was regarded as inappropriate for particle fueling. As a consequence, all major tokamak experiments converted their pellet launch systems to allow for high-speed launch from the HFS in order to achieve maximum pellet particle deposition depths resulting in an optimized refueling behavior. Often this optimization was done even at the expense of sacrificing the option to launch from the LFS.

Originally considered as an unwanted negative effect that just hampered pellet particle refueling, the ELM triggering by the pellets at last was realized to bear helpful aspects as well. With growing tokamak size the ELMs become a severe threat for the in-vessel components. For the largest present day machines like JET, ELMs can cause target ablation in the divertor; ${ }^{7}$ for ITER the undamped intrinsic ELMs would most probably result in a fatally fast erosion of the divertor plates when operating in the foreseen operational scenario. ${ }^{8}$ Hence, strategies are under investigation aiming to abandon or at least mitigate the ELM impact. It turns out that a rather promising approach is the enhancement of the ELM frequency $\left(f_{\mathrm{ELM}}\right)$.

In JET and ASDEX Upgrade, if the total energy of the plasma equaled its thermal energy content the following relation was found: $f_{\mathrm{ELM}} \times \Delta W_{\mathrm{ELM}}=0.2 \times P_{\text {loss }} \quad\left(\Delta W_{\mathrm{ELM}}\right.$ plasma energy loss induced by the ELM; $P_{\text {loss }}$ : power lost to the divertor). ${ }^{9}$ Hence, as the total energy carried by all the ELMs remains roughly constant with increasing $f_{\mathrm{ELM}}$, as $\Delta W_{\mathrm{ELM}} \sim 1 / f_{\mathrm{ELM}}$ the ELMs become mitigated. Several techniques have been investigated so far for their ability to drive 
$f_{\text {ELM }}$ beyond its intrinsic value, some of them successful. However, any approach for ELM pacing has to prove not just its ability to establish control of $f_{\text {ELM }}$ but must also inevitably prove the technique easy to handle, without imposing operational hazards and with unwanted side effects remaining at an acceptable level. Certainly, injection of cryogenic pellets meets all these criteria. Its technology is at hand, well developed, and without operational hazard, while, by using fuel particles, no impurity contamination of the plasma takes place. Dedicated investigations have already demonstrated that pellet ELM pacing can establish external control of $f_{\text {ELM }}$ and ELM mitigation with bearable side effects. ${ }^{10}$ Pelletbased ELM frequency control has already become a part of the toolkit for plasma control. ${ }^{11,12}$ This control is accompanied by a mild loss of the plasma confinement, most probably due to pellet fueling still being present. A relation for the plasma energy $W \sim f_{\mathrm{ELM}}^{-0.16}$ was found for the pellet pacing, ${ }^{10}$ significantly less than the $W \sim f_{\mathrm{ELM}}^{-0.6}$ observed for constant heating power in the case of intrinsic ELMs. ${ }^{9}$ Indeed, all these investigations were performed using pellet launcher systems optimized for fueling rather than for edge control purposes.

It was realized that there is still ample headroom for operational enhancement once more appropriate pellet parameters can be applied in order to avoid fueling not being required. Moreover, a system adapted for edge control yields the option to perform more suitable experiments aiming to uncover the underlying physics and dynamics of ELMs. Consequently, as the requirements for pellet launcher systems devoted to plasma particle refueling are quite controversial compared to those for ELM pacing, and the available ASDEX Upgrade pellet injector was fully optimized for the refueling task, ${ }^{13}$ a new system was developed entirely dedicated for ELM control and ELM investigations.

\section{REQUIREMENTS FOR THE SYSTEM}

Creating a new, additional pellet system promised an enhancement of the operational features in ELM control scenarios since fueling and hence convective losses are reduced. Moreover, higher pellet and hence higher ELM frequencies can further reduce the ELM size. And finally, dedicated experiments making use of a lower pellet velocity and more flexibility of the injection geometry could allow for a better understanding of the ELM physics. The task was therefore to develop a new system adapted for edge control as a supplement to our fueling system used so far for pellet ELM triggering. Requirements for the system are prescribed by the needs for ELM control obeying the constraint of minimizing unwanted side effects, i.e., fueling, but also keeping in mind the intention to enable dedicated ELM investigations. Further on, to minimize risks and costs, we wanted to stay within the range of available technology as much as possible. Of course reliable operation with a high pellet delivery efficiency $(\varepsilon \equiv$ pellets delivered/pellets requested) has to be guaranteed. In principle, we aimed at the highest possible pellet frequency with the smallest possible particle flux.

We know from our previous experiments that a pellet $N_{P}$ of only about $10^{18} \mathrm{D}$ atoms would be sufficient to trigger
ELMs in a typical ASDEX Upgrade H-mode discharge. As this is on the order of about $1 \%$ of the pellet sizes used so far, the particle flux could potentially be reduced to an ignorable amount. However, such a drastic reduction of the pellet size would call for a pellet diameter on the order of $250 \mu \mathrm{m}$. Production, handling, and transfer of submillimeter-size cryogenic pellets is beyond our experience. Submillimetersize pellets are hard to produce, as pellets stemming from an extruded ice rod become more fragile with increasing surface/volume ratio. As well, transfer losses due to ice sublimation have to be anticipated. In order to guarantee a sufficient pellet delivery efficiency we have chosen a circular pellet cross section of $2 \mathrm{~mm}$ and set the length to $1 \mathrm{~mm}$, according to the technical parameters of the available extrusion cryostat. Most probably with these pellet parameters, size and thus particle flux optimization are not yet fully achieved. We therefore envisage exchanging the current extrusion cryostat system for a new one with a reduced pellet cross section (different diameters optional) as a next step.

The pellet velocity was chosen to lie in the range of about $200 \mathrm{~m} / \mathrm{s}$.

This comprises a speed low enough to avoid deep penetration and hence strong fueling, but high enough to keep control of pellet trajectory since repulsion from ablating gas can cause strong deviation for slow pellets. As well, pellet flight times from the accelerator to the torus through the several-meters-long guiding tubes should last no more than some $10 \mathrm{~ms}$, or else they might impose response times too long for a meaningful control approach. Thus, we chose the blower gun principle ${ }^{14}$ for pellet acceleration relying on the viscous drag acceleration. ${ }^{15}$ If hydrogen is used as the driver gas no problem is raised by the gas reaching the torus. The option to reduce the pellet velocity $v_{P}$ by using a driver gas with a lower sound velocity can be used as well if the parasitic driver gas flux to the torus is kept sufficiently low.

Since experiments performed so far still suffered from an insufficient available pellet rate, ${ }^{12}$ for the pellet repetition rate $f_{P}$ at least $100 \mathrm{~Hz}$ were prescribed. Such a frequency was thought already sufficient to establish ELM frequency control with a reasonable ratio of driving frequency $f_{P}$ to initial intrinsic ELM frequency $f_{\mathrm{ELM}}^{0}$ for type-I ELMy $H$-modes discharge scenarios typically investigated at ASDEX Upgrade including radiative mantle experiments. ${ }^{16}$ It turned out that, to meet this requirement, pellet extrusion and acceleration have to be processed in parallel. A new scheme was therefore developed, called the tic-tac shuttle.

The new system is intended to be operated in addition to the already existing one injecting from the HFS; the design was laid out for LFS launch. For this scheme ELM pacing is expected to show a better performance anyway; additionally, experiments comparing HFS and LFS pacing become possible using both systems. Two different injection lines are installed for the new system; they can be altered between plasma discharges. The first one is set up with a straight injection path, dedicated for operational use aimed at simplicity and hence high reliability. The alternative one has a tilted injection path and aims at dedicated physics investigations relying on extremely shallow particle deposition and vanishing radial pellet velocity by creating a tangential point 
TABLE I. Features and parameters for both pellet launch systems at ASDEX Upgrade.

\begin{tabular}{ccc}
\hline \hline System task & $\begin{array}{c}\text { Fueling } \\
(\text { old })\end{array}$ & $\begin{array}{c}\text { ELM triggering } \\
\text { (new) }\end{array}$ \\
\hline Injector type & Centrifuge & $\begin{array}{c}\text { Blower gun } \\
\text { Shallow }\end{array}$ \\
Particle deposition & Deep & LFS straight \\
Launch position & HFS tilted $\left(72^{\circ}\right)$ & LFS tilted $\left(33^{\circ}\right)$ \\
& & 1.6 \\
Pellet size nominal $\left(10^{20} \mathrm{D}\right)$ & $1.7-4.3$ & $100-350$ \\
Pellet velocity $(\mathrm{m} / \mathrm{s})$ & $240-1000$ & 143 \\
Pellet rate max. $(\mathrm{Hz})$ & 62 & \\
\hline \hline
\end{tabular}

of the pellet path with respect to the plasma flux surfaces in the plasma edge region or the scrape-off layer (SOL).

A comparison of the main features and parameters for the old fueling-optimized system and the new system for ELM pacing is compiled in Table I.

\section{LAYOUT OF THE SYSTEM}

An overview of the experimental setup is given in Fig. 1. The right part shows schematically the new blower gun injector. Pellets are delivered from the injector to the torus entrance port via a $5 \mathrm{~m}$ long Teflon guiding tube with $6 \mathrm{~mm}$ inner diameter. Directly outside the entrance valve the pellets can be connected to one of the available injection lines (horizontal only at present).

The left part (inside the frame) shows a poloidal cross section of the tokamak ASDEX Upgrade in the 2006 configuration with an almost accomplished transfer to a full tungsten (W) first wall. ${ }^{17}$ The presently available injection line aims at direct and straight launch into the plasma slightly below the torus horizontal midplane. This setting is considered most easy to handle and is thus designated for operational oriented experiments. Results reported regarding first pellet injection tests into ASDEX Upgrade were obtained using this setting. Alternatively, a transfer tube is under construction aiming to inject at an angle tilted by $33^{\circ}$ with respect to the horizontal plane. In this investigation set-

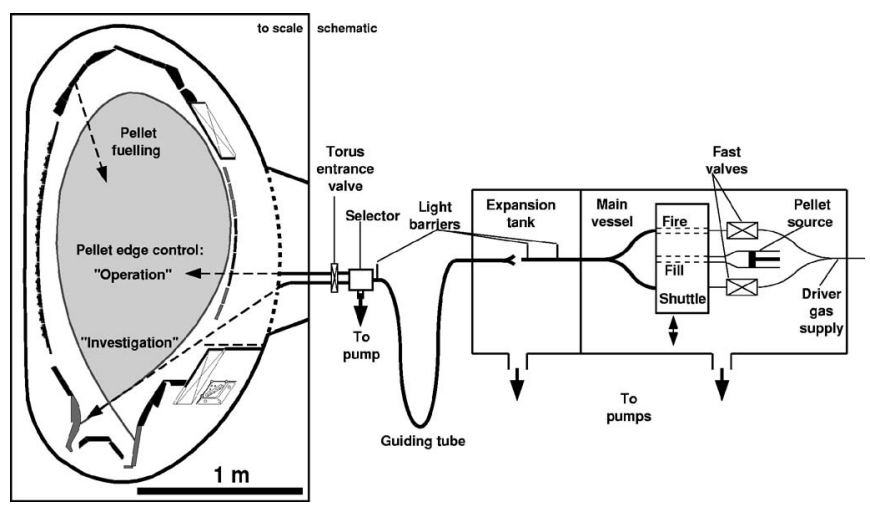

FIG. 1. Overview of the experimental setup. Left (part in box to scale): Poloidal cross section of the tokamak ASDEX Upgrade in the 2006 configuration (W-coated wall elements in black, $\mathrm{C}$ in gray) with a typical plasma configuration foreseen for ELM investigation experiments; all pellet injection paths: horizontal (existing) and tilted (planned) LFS launch with new system, fuelling trajectory (operational). Right (schematic only): Blower gun injector delivering pellets to the torus, at the torus entrance port the connection to the selected injection line is established. ting pellets will tangentially transfer through the edge plasma region close in or outside the last closed magnetic surface (separatrix). In Fig. 1 a typical plasma configuration foreseen for the ELM investigation experiments is given with a designated pellet tangent at the separatrix. It is expected that pellets will show only extremely shallow penetration before they possibly impinge on the inner divertor leg. Laboratory tests showed that pellets in the applied speed and size range are harmless to the carbon tiles; the parasitic gas should be effectively trapped in the divertor region and removed by the pumps.

In the following, a more detailed description of the main components will be given.

\section{A. Pellet source}

The pellet source comprises two cryostat systems, one for extrusion and one for storing the ice. Liquid helium $(\mathrm{He})$ is used for cooling; the total consumption is about $5 \mathrm{l} / \mathrm{h}$. Every cryostat has its own coolant and temperature control system, so extrusion and storage temperatures can be chosen and adjusted separately. The D fuel (usually high-purity D is used, but alternately slightly doped samples using, e.g., a few percent of neon tracer material can be used with the same settings; $\mathrm{H}$ can be processed as well, but requires an adaptation of the temperature and pressure parameters) is delivered from a storage volume to the extrusion cryostat's freezing cell. Small ice crystallites are produced at pressure of about 2 bar and a temperature of 5-10 K. Before extrusion is started, compression and annealing by a tempering heat pulse lasting for about $20 \mathrm{~s}$ is applied, this was found to improve ice quality and pellet stability. Finally, at a sample temperature of $15 \mathrm{~K}$ ice extrusion at an averaged extrusion speed of about $1 \mathrm{~mm} / \mathrm{s}$ is performed through a circular nozzle with $2 \mathrm{~mm}$ diameter by applying a pressure of 14 bar on the piston (diameter $6 \mathrm{~mm}$ ). The ice rod is extruded into the storage cryostat (circular $90^{\circ}$ bent); the length of the single storage channel and hence of the stored ice rod is $124 \mathrm{~mm}$. A light barrier mounted at the bottom of the storage cryostat indicates filling is finished and stops extrusion. The ice reservoir can be kept there if required for up to $1 \mathrm{~h}$ at a temperature of about $5 \mathrm{~K}$. If a pellet request arrives, the ice rod is shifted out at the bottom of the storage cryostat by a mechanical lever. The single step width is $0.5 \mathrm{~mm}$. Every step requires $1 \mathrm{~ms}$; after the shift a pause of $2 \mathrm{~ms}$ is required to bring the vibrating ice rod to rest. Within this boundary conditions, any sequence is accepted; it is, e.g., possible to interrupt a feeding/ pellet sequence and/or change the feeding/pellet rate.

\section{B. Acceleration unit}

The main part of the acceleration system is the shuttle, which makes parallel pellet feed and acceleration along two lines in turn possible, allowing us to reach high repetition rates. The shuttle consists of an aluminum frame with an inlay of Vespel and two permanent magnets at both ends. It is mounted movably on a slide and driven to the end positions by two coils, one pushing and the other one pulling and capturing the shuttle in turn. Fixation at the alternating positions is supported by the two permanent magnets. Two small 
bores of $\varnothing 2 \mathrm{~mm}$ are provided in the shuttle to accept the pellet, bringing it to the starting position for the acceleration, and acting as parts of the barrel. With the shuttle in an end position, one of the bores is located in front of the storage cryostat's exit and can be filled from the ice rod. Pellet cutting from the rod is achieved when the shuttle moves over to the alternate position. The bore just filled now arrives in its firing position while the other one is shifted from its firing position to the common filling position. As can be seen from the schematic drawing of the unit in Fig. 1, the common filling position opposite the extrusion cryostat (drawn on the same side in Fig. 1 for simplicity) bottom is located between the firing positions opposite the two gas valves. In its firing position, the filled caliber completes the acceleration barrel now consisting of the driver pipe at the top of the gas valve nozzle, the caliber, and the onset of the guiding tube. The accelerating gas pulse is initiated by a short opening $(1.2-1.5 \mathrm{~ms}$, slightly depending on the pressure in the propellant gas reservoir) of the fast valve. The gas streaming from the nozzle reservoir into the evacuated multisegmented barrel causes viscous drag acceleration ${ }^{15}$ of the pellet to a velocity that is, on average, significantly below the gas sound speed. Preselecting the appropriate driver gas's species and pressure yields acceleration to the requested $v_{P}$ value. The two initial parts of the guiding tube open out finally into the single main tube after $33 \mathrm{~cm}$. To avoid excessive pellet evaporation (especially at low repetition rate operation; when interrupting a sequence the last pellet remains in the shuttle) the shuttle is cooled. However, too-strong cooling can have an adverse impact on the shuttle's gentle, smooth, and precise motion. Best overall performance was achieved at a shuttle temperature of about $85 \mathrm{~K}$. Even with the shuttle thus operating at a temperature quite far beyond the sublimation temperature of the $\mathrm{D}$ pellet (about $18 \mathrm{~K}$ in the pressure range), the Leidenfrost effect ${ }^{18}$ avoids too-fast pellet evaporation and resultant unacceptably high gas loads.

\section{Pumping and transfer system}

Although the Leidenfrost effect reduces the release of gas by pellet sublimation, ablating pellet material and the driving gas can form a severe threat to stable operation. The gas pressure in the main vessel has to be kept below $0.1 \mathrm{~Pa}$ to prevent the onset of significant convective heat flux to the cryostat systems. Once the heat load carried by the exhaust gas approaches a critical limit, strong evaporation from the stored ice rod sets in, leading into a vicious circle of further enhancing the exhaust gas pressure, increasing in turn the heat flux even more. Then, finally, temperature destabilization and a rapid loss of the ice inventory take place. Keeping the main vessel pressure sufficiently low even in the case of very low pellet repetition and/or for frequently interrupted pellet sequences, a combined approach relying on strong pumping and sealing off the gas/tube system was formed. Some restrictions exist for the sealing of the gas system at the multisegmented acceleration barrel. Complete tightening of the main vessel near the shuttle imposes unacceptably high damping of the motion. So, a certain amount of leaking gas escaping into the main vessel body is inevitable and has to be removed by sufficient pumping. This is achieved by two turbo molecular pumps each of $360 \mathrm{l} / \mathrm{s}$ pumping capability mounted at separate main vessel ports. Additional pumps of the same type are employed for evacuation of the expansion tank and the selector (one at each). The main fraction of driving gas already can be separated and exhausted via the expansion tank before sending the pellet into the main part of the ex vessel guiding system. Remains of the driver gas flowing into the guiding tube and gas released from the pellet body along its passage are scraped off in the selector unit, which acts as a second expansion tank as well. The pellet guiding system is composed of Teflon tubes of $\varnothing 6$ $\mathrm{mm}$ diameter. The main ex vessel section, spanning from the expansion tank to the selector, is $5 \mathrm{~m}$ long and forms a rather high resistivity for any parasitic gas flow. The torus entrance valve forms a mechanical, vacuum, and electrical barrier between the torus vessel and the pellet system. It is kept closed unless gated open during an injection sequence, provided that the torus protection system ${ }^{19}$ checking all relevant system parameters validated this request. Tubes inside the torus located in sector (octant) 5 are made from stainless steel. The straight "operation" tube launches the pellets at torus coordinates $R=2513 \mathrm{~mm}, z=-115 \mathrm{~mm}$. Once the alternative track becomes available, distribution of the pellets to one of the injection lines is achieved by a mechanical switch in the selector. The setting can be changed between two plasma discharges.

\section{Intrinsic and test bed diagnostics}

The pellet system is equipped with numerous permanent intrinsic diagnostics; several additional ones have been used during the operation in the test bed. Temperature sensors embedded in the cryostat systems and the shuttle allow for control and supervision of pellet production and storage. A conventional video system is employed for the observation of ice extrusion, providing a quality control of the ice rod.

For characterization of the system the main pellet parameters $N_{P}, v_{P}$, and $\varepsilon$ are measured. Two light barriers performing a time-of-flight measurement determined the initial $v_{P}$ just after the acceleration. Additional light barrier(s)at the end of the main ex vessel transfer tube (a single one at the torus, two for a full local $v_{P}$ measurement in the test bed) acted as pellet flyby monitors. Pellet integrity determination and measurement of $N_{P}$ in the test bed were done both at the begin and the end of the main transfer tube by laser flash shadowgraphs. Pictures obtained could be used to find out the status of every single pellet (intact, damaged, or destroyed) reliably, but due to the two-dimensional image, averaging over a set of pellets is required for a useful mass measurement. Using sequences of typically 10 pellets, the estimated accuracy is about $20 \%$ of the initial pellet mass. An additional value for the transferred pellet mass was derived by measuring the released gas amount when firing and destroying pellets in a test bed target tank with calibrated volume. In this case, the accuracy was higher than for the shadowgraphs, estimated to be about $10 \%$. Besides indications from shadowgraphs and different light barriers, successful pellet arrival was detected by an acoustic impact shock sensor with high accuracy. After the transfer of the pellet injection system to the torus hall the operation of sev- 
eral intrinsic diagnostics was not longer possible. However, for injection experiments the well-diagnosed ASDEX Upgrade plasma ${ }^{20}$ itself provides powerful diagnostics.

\section{E. ASDEX Upgrade}

ASDEX Upgrade is a midsize tokamak with torus radius $R_{0}=1.65 \mathrm{~m}$, minor plasma radius $a_{0}=0.5 \mathrm{~m}$, and plasma volume $V_{\text {plasma }}=13 \mathrm{~m}^{3}{ }^{21}$ Its design combines the successful divertor concept with the requirements of a next-step fusion device, in particular the need for an elongated plasma shape and poloidal magnetic field coils outside the toroidal field coils. Since 1999, the tungsten coverage of the wall surface has progressively been increased and is foreseen to be completed for the next campaign in 2007; the current status can be seen in Fig. 1. Recent operational experience with an almost-full tungsten first wall showed that sufficiently high ELM frequency must be maintained to clamp the impurity content; ${ }^{22}$ otherwise, impurity accumulation causes a radiative plasma collapse. Hence, impurity control tools like pellet pacing become of vital importance. ASDEX Upgrade is equipped with a sophisticated protection, safety, and control system $^{19}$ in which the new pellet system is included to make experiments feasible where pellet pacing is applied as an additional control tool.

In turn, the control system enables the performance of dedicated physics investigations applying plasma shaping and position control features in order to achieve a variation of pellet-induced deposition profiles with a fixed injection trajectory by moving the plasma column accordingly. Utilization of this approach's full potential is still to come. Just simple feed-forward injection in a quiescent stable and robust plasma discharge type was performed for the first phase, dedicated to bringing the new launcher into operation and performing experiments for system characterization.

In this approach we made use of the plasma for monitoring the impact of pacing on the ELM rate. The $D_{\alpha}$ radiation emitted from the pellet and its surrounding region acted as pellet monitor. Cameras measure the pellet trajectory and penetration depths. ${ }^{23}$ The impact of the pellet injection on the electron density profile was measured by a DCN laser interferometer.

\section{RESULTS FROM TEST BED}

The main intention of the test bed operation was to characterize and optimize the operational parameter range. Main parameters of interest were pellet particle inventory $N_{P}$, pellet velocity $v_{P}$, pellet repetition rate $f_{P}$, and pellet delivery efficiency $\varepsilon$. The initial $N_{P}=1.6 \times 10^{20} \mathrm{D}$ is already set by the hardware. This mass is known to be more than sufficient for ELM triggering in a typical ASDEX Upgrade type-I ELMy $H$-mode; thus, it just had to be ensured that mass losses occurring during pellet acceleration and transport were not causing pellet disintegration due to strong erosion.

Acceptable operation requires an $\varepsilon$ value fairly close to unity. On the other hand, the maximum achievable $f_{P}$ is set by the construction boundary conditions. It turns out that $v_{P}$ is the only remaining free parameter to choose/adjust. In general, this can be done for the applied approach by altering

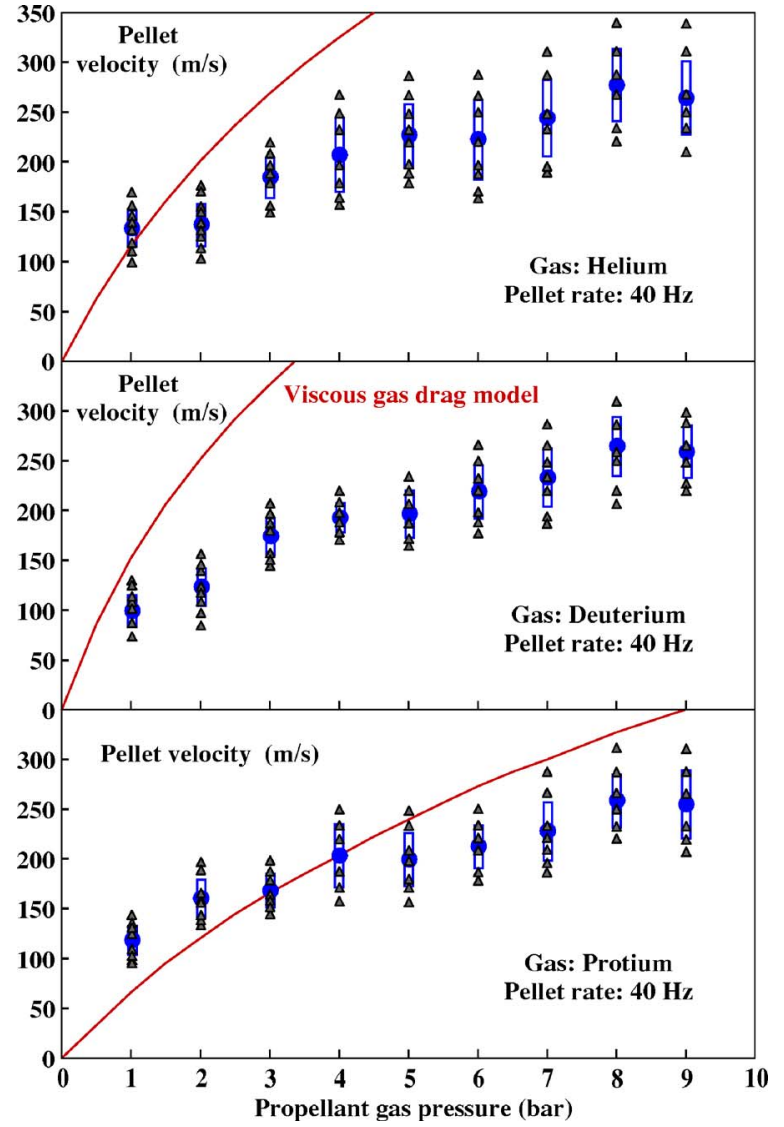

FIG. 2. Pellet velocity vs propellant gas pressure for using helium (upper), deuterium (middle), or protium (lower) driver gas. All pellets fired in sequences at a repetition rate of $40 \mathrm{~Hz}$. Triangles represent data of the single pellets, filled circles their average. Pellet velocities calculated by the viscous drag acceleration model (Ref. 15) for the given setup are given by the solid curves.

the driver gas pressure, the driver gas species, or the injection geometry parameters like barrel length or the diameter ratio of pellet/barrel. ${ }^{15}$ Of course the latter two are already fixed by the setup; only the first two can be used for $v_{P}$ adaptation.

As driving gases we used ${ }^{1} H,{ }^{2} H(\mathrm{D})$, or $\mathrm{He}$, performing pressure scans of the driver gas in the reservoir from 1 to 9 bar. Results are shown in Fig. 2, where for the three different driver gas species achieved $v_{P}$ values are plotted versus reservoir pressure. In all cases, pellets were fired in sequences of $f_{P}=40 \mathrm{~Hz}$. Data recorded for single pellets are represented by triangles, and the average of the data sets by the filled circles. As expected, the pellet velocity can be increased by rising the propellant gas pressure. By varying the pressure applied, speed values ranging from below $100 \mathrm{~m} / \mathrm{s}$ to peak values reaching almost $350 \mathrm{~m} / \mathrm{s}$ can be achieved. Any of the three investigated driver gas species can be used to establish a pellet velocity around $200 \mathrm{~m} / \mathrm{s}$, thought suitable for the envisaged experiments. Since pellet speed depends only weakly on the propellant gas pressure in this regime, stable operation can be achieved quite easily. For our experimental approach this is quite helpful as this eases the choice of the driver gas — hydrogen can be used to prevent plasma dilution, or helium for safety reasons. Advantageous for operation, this almost canonical behavior of the pellet velocity 
versus propellant gas pressure is a bit surprising. This becomes clear by comparison to simple code calculations of the pellet acceleration based on the viscous drag acceleration model as discussed in Ref. 15. Following Gilliard et al.'s analysis of a very similar gun structure, we adopted a drag coefficient, $c_{D}=0.7$. For the gas velocities we took $u=1000 \mathrm{~m} / \mathrm{s}$ for $\mathrm{D}$ and $\mathrm{He}$ and $u=1300 \mathrm{~m} / \mathrm{s}$ for protium. Adiabatic/isentropic gas expansion is assumed, taking $p \times V^{\kappa}=$ const $(p=$ gas pressure, $V=$ gas volume); the adiabatic coefficient $\kappa=c_{p} / c_{V}$ is the ratio of the specific heat capacity at constant pressure and constant volume; it is approximated by $\kappa=f+2 / f$ with $f$ degrees of freedom, i.e., $\kappa=7 / 5$ for the hydrogen isotopes and 5/3 for helium. Finally, material parameters (gas and pellet density, pellet diameter) and the geometric properties of our injection setup (barrel length and diameter, gas reservoir volume) completes the set of model parameters-leaving no free-adaptation variable. Results obtained by the calculations are included for each gas species in Fig. 2 as solid curves. Despite its higher gas speed, protium is expected to yield the slowest pellet velocities because its mass density is about half the corresponding values of deuterium and helium. For helium and deuterium, gas densities and speed are almost identical; deuterium should yield stronger acceleration due to the smaller $\kappa$ value representing the higher internal energy stored in the D-D bond. Comparing experimental values with the calculations, there is good agreement with respect to the absolute value of the pellet speed-ranging from about $10 \%$ to $30 \%$ of the gas velocity ( $\approx$ sound velocity). For protium there is also a reasonable agreement of the pressure dependence of $v_{P}$; for deuterium and helium this is clearly not the case. In particular, there seems to be a restriction in the maximum accessible pellet velocity. A possible reason for this might be the increasing friction inside the barrel. Although the full acceleration behavior is not fully reproduced by the viscous gas drag model, it seems to describe the driving mechanism quite well. A more accurate modeling needs additional data from more sophisticated test runs employing as well further internal data, e.g., on the time evaluation and the spatial distribution along the tube of the gas flow velocity and density in the acceleration tube; these are not available because of the robustness of the system. However, as a satisfactory operational parameter range was realized, we refrained from further efforts dedicated to this aspect.

When approaching the highest reasonable possible pellet repetitions rates, the resulting pellet velocities for a given gas configuration start to shrink. The achieved $v_{P}$ values for a given gas parameter set (He propellant gas at 3 bar) plotted versus the preselected $f_{P}$ value are shown in Fig. 3 .

Obviously, premature return of the shuttle already begins to restrict the duration of the applied real gas pulse by scraping off more and more of the gas pulse tail. With the gas pulses becoming shorter and shorter, significant parts of the driver gas have already passed the pellet before it reaches the end of barrel. Hence, this velocity degradation is because the effective acceleration time and hence the effective barrel length start to shrink. The highest $f_{P}$ value where the system

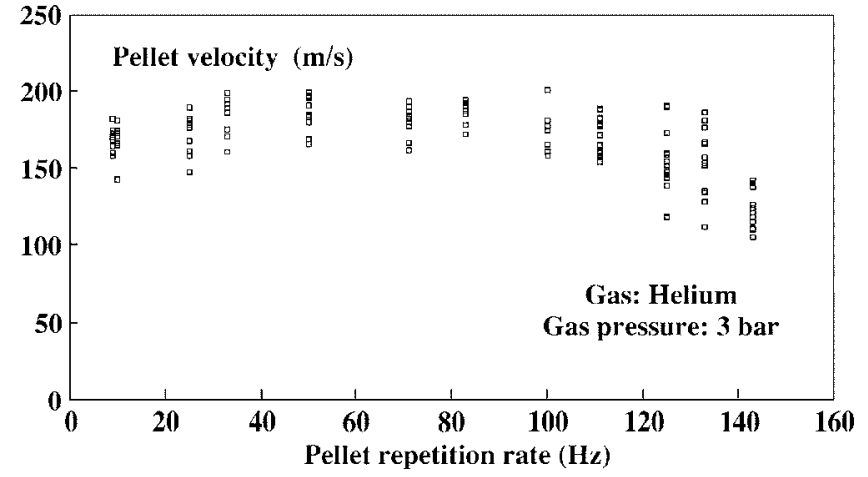

FIG. 3. Pellet velocity vs pellet repetition rate (helium driver gas at 3 bar). Beyond $110 \mathrm{~Hz}$ a mild reduction in velocity sets in due to shortening of the acceleration gas pulse.

still can be reasonably operated was chosen to be $1 / 7$ $\times 10^{-3} \mathrm{~s} \approx 143 \mathrm{~Hz}$, despite the mild but still acceptable reduction in velocity.

Besides the pellet velocity, the reliability is affected as well by increasing $f_{P}$. This is visualized in Fig. 4, where $\varepsilon$ is plotted vs $f_{P}$ for the same gas configuration as used in Fig. 3. There is some very mild degradation observed for the delivery efficiency above $f_{P}=110 \mathrm{~Hz}$ from about 0.9 to about 0.8 . Shortening the pellet firing sequence cycle imposes some loss of the driving forces required for the acceleration; this might result in pellet loss. In addition, pellet damage or even destruction might take place in some cases during the feed-in process, since the pellet is not yet in position or at rest when cutting or acceleration is initiated.

In order to allow a quality control of the pellets, shadowgraphs using laser flash illumination to avoid movement blur were taken. Two photo stations, one at the exit of the acceleration unit and the other one at the exit of the main ex vessel tube, were installed. Investigation of pellet integrity showed, as already indicated by the reliability data within the operational boundaries envisaged (driver gas pressure $<10$ bar, $f_{P} \leq 143 \mathrm{~Hz}$ ) there are no disintegrated pellets. Especially, there is no impact on this result by the orientation of the pellet at the exit of the acceleration unit. Obviously, pellets already start to totter in the initial part of the guiding tube. At the exit of the acceleration sections pellet bodies can be observed in any orientation with respect to the flight di-

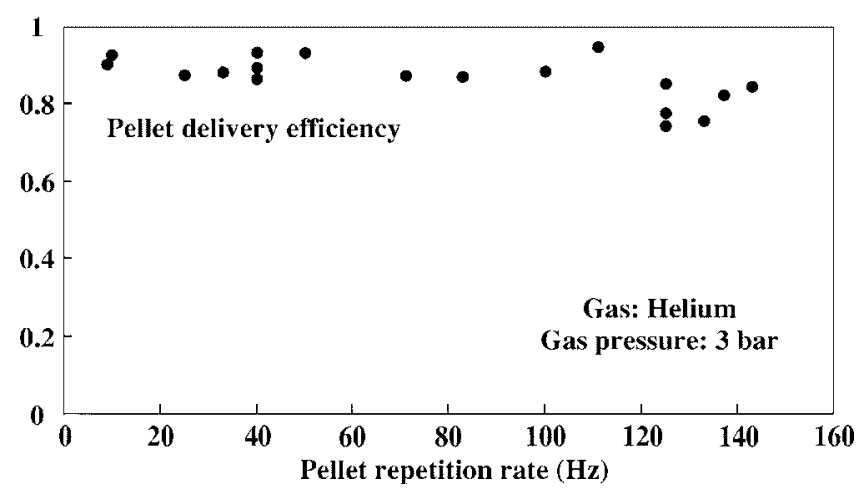

FIG. 4. Pellet delivery reliability vs pellet repetition rate (helium driver gas at 3 bar). Beyond $110 \mathrm{~Hz}$ a very mild reduction sets in, like for the pellet velocity. 


\section{Before transfer tube}
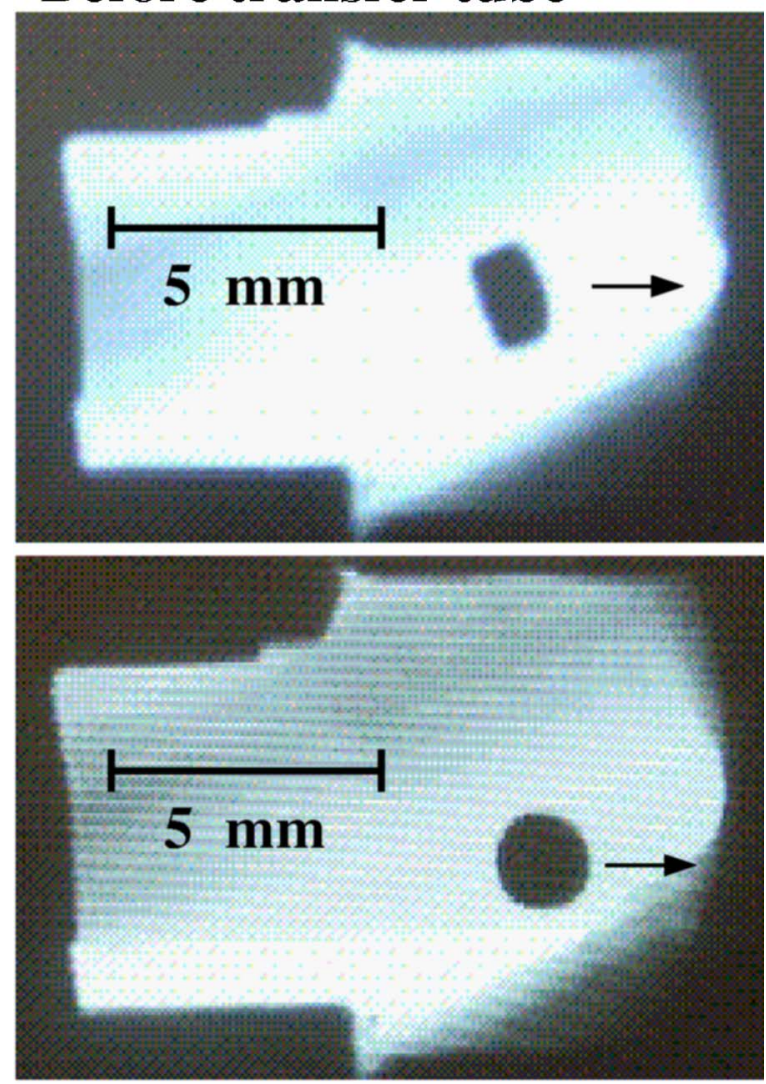

\section{After transfer tube}

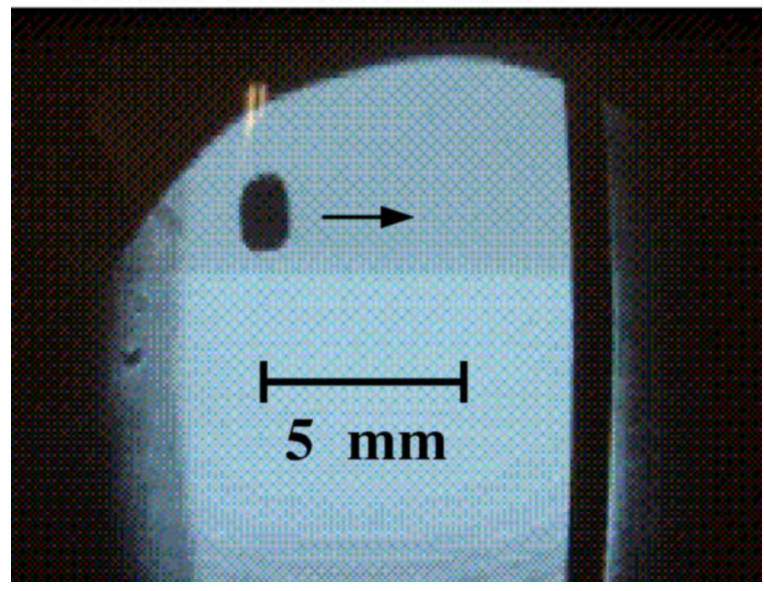

FIG. 5. Pellet snapshots taken by a shadowgraph system at exit of acceleration section inside expansion tank (upper and middle) and at the exit of the main ex vessel guiding system (lower) after transfer through a $5 \mathrm{~m}$ long tube.

rection. This can be seen in Fig. 5, where the upper two frames show pellets at the barrel exit, the upper one oriented with its circular cross section almost perpendicular, the middle one with its circular cross section parallel to the flight direction (arrow). At the exit of the main ex vessel tube almost all pellets arrive with the same shape, just showing slight indications for erosion. An example is shown in the lower part of Fig. 5.

To characterize the mass degradation, we measured the arriving particles in the test bed tank and compared it with the calculated initial pellet mass assuming a perfect shape. It

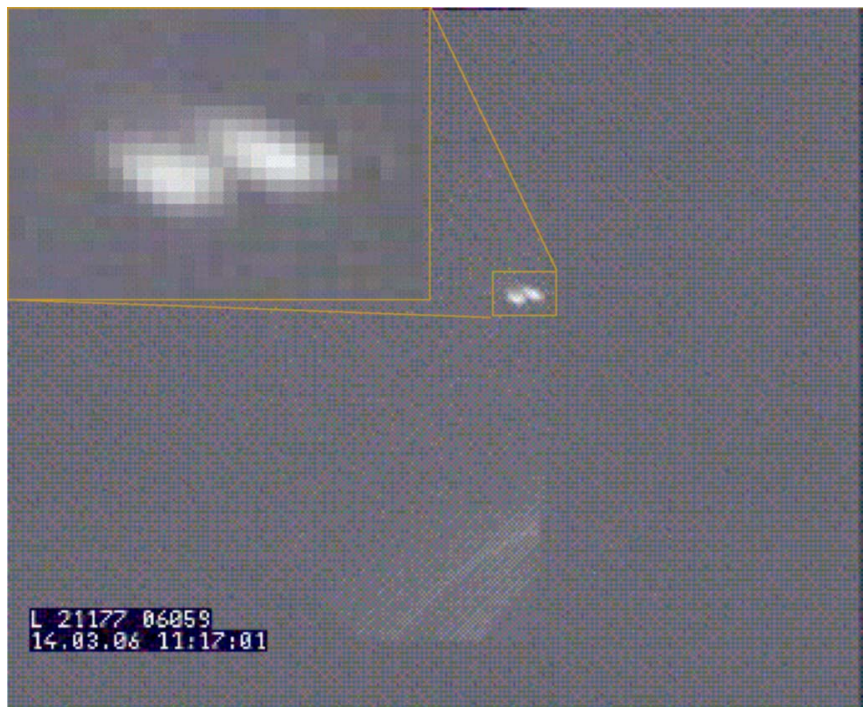

FIG. 6. Video recording (half frame, exposure time $20 \mathrm{~ms}$ ) of two pellets launched at $100 \mathrm{~Hz}$ repetition rate into an ongoing ASDEX Upgrade plasma discharge.

turned out roughly $2 / 3$ of the nominal mass arrived in the tank, so total transfer losses clearly beyond the accuracy of our mass measurements were found with estimated approximately $30 \%$ in a range as expected from investigations performed with our fueling system. ${ }^{24}$

\section{FIRST APPLICATION AT ASDEX UPGRADE}

At the successful end of the test bed campaign, the range of operational system parameters was fixed and the system thereupon validated for operation at ASDEX Upgrade. Transferred to ASDEX Upgrade and integrated into its safety and control system, the very first tests of pellet injection and ELM pacing with the new launcher system are reported here. Pellet injection was performed on a piggyback basis during $H$-mode phases developing low ELM frequencies. To attempt a further reduction of fueling, the pellet length was reduced to $0.5 \mathrm{~mm}$. Faultless operation of the entire system is demonstrated by the successful arrival of sound pellets in the plasma. An example is shown in Fig. 6, displaying a half frame (exposure time $20 \mathrm{~ms}$ ) extracted from the torus wideangle video recording system. Two ablation clouds from pellets launched during a $f_{P}=100 \mathrm{~Hz}$ train can be seen close to the designated intersection point of pellet trajectory and plasma separatrix. From the homogeneous shape of both ablation clouds (blowup displayed as inset) and the measured penetration depths and density as well, increased intact pellet arrival can be concluded.

Although our tests have proven the new system is essentially working, we found its reliability to deliver intact pellets significantly smaller (30\%) than in the test bed (90\%). This was most probably due to insufficient pellet stability for a cylindrical body with a diameter/length ratio of 4 .

Approaches to enhance this during an in situ commissioning and also to revert the length reduction came to an end with the abrupt, premature stop of the AUG 2006 campaign. Instead, we have now installed an enhanced video stand at the torus entrance port and commenced commission- 


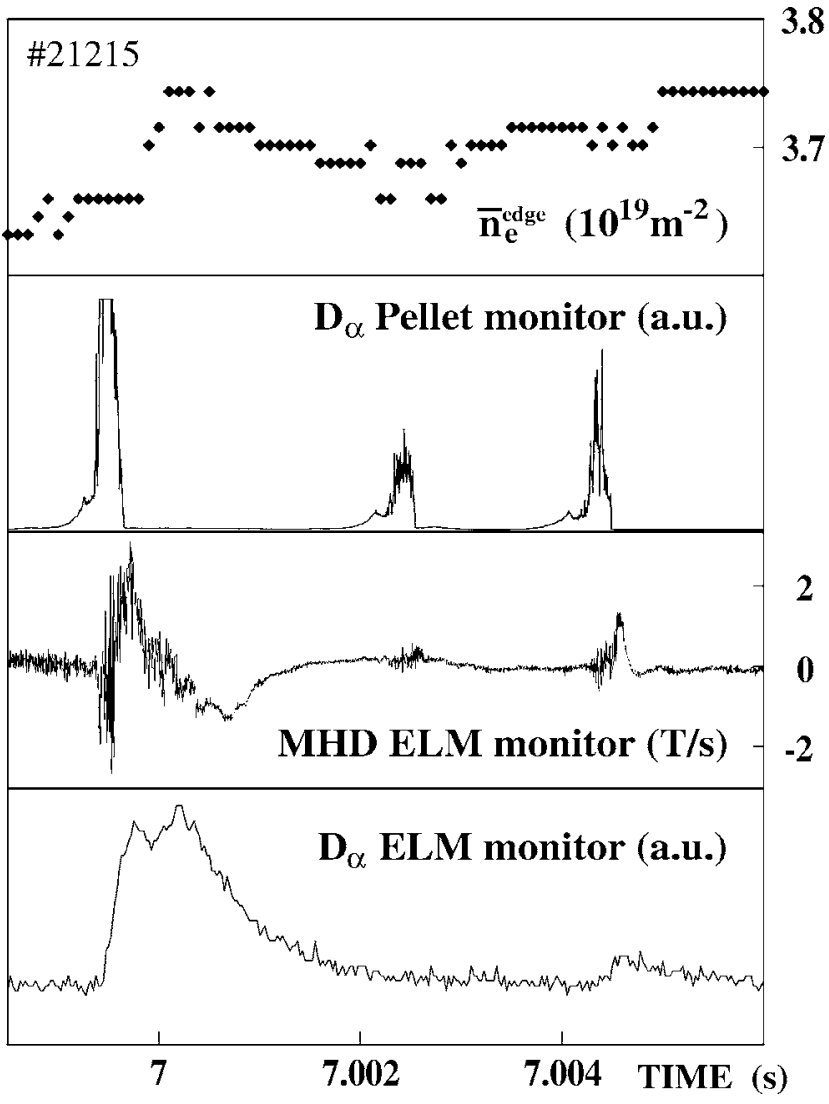

FIG. 7. First preliminary results from ELM trigger investigations. Sequence of three pellets of presumably different size (note saturation of pellet monitor for first pellet). Different pellet mass can result in changed ELM characteristics. First (largest) pellet triggers a clear ELM and imposes significant plasma perturbation, third (medium size) just triggers an ELM, second (smallest) fails to produce full-size ELM.

ing. Our aim is to have the system with full performance ready at the start of the 2007 campaign. Currently a new cryostat system is under construction, designed for smaller cylindrical pellets with $\varnothing=1 \mathrm{~mm}$ and length $0.5 \mathrm{~mm}$, aiming for a significant reduction of the fueling impact.

Despite pellet fragmentation and destruction hampering the first short experimental approach, interesting physics investigations on ELM triggering already could be performed. One example is shown in Fig. 7, displaying the impact of three pellets injected during a type-I ELMy $H$-mode phase. Obviously, as indicated by the pellet ablation $D_{\alpha}$ monitor and the measured line-averaged density along a line of sight through the plasma edge region, pellets had different sizes. The first and presumably largest pellet (note saturation of the pellet monitor) clearly triggers a strong ELM and also causes significant perturbation of the background plasma [indicated by the strong pressure pulse to the outer divertor displayed by the $D_{\alpha}$ ELM monitor and the magnetohydrodynamic (MHD) perturbation, both lasting about $2 \mathrm{~ms}$ ]. The last pellet triggers an ELM still clearly visible on both monitor signals, but with an almost negligible perturbation of the plasma. In this respect, the mass of this pellet can be considered as ideal for pacing purposes. The pellet in between has still some impact on the MHD behavior but fails to release a full-size ELM. This can be a first hint that pellets that are too small and/or not sufficiently deep penetrating cannot serve as ELM pacer. It is understood that the first results shown here are not yet sufficient for drawing reliable conclusions, but very nicely demonstrate already the potential of the new injector.

\section{OUTLOOK}

In this article we presented first results and operational features achieved with a new pellet launcher system designed and developed for ELM pacing and corresponding physics investigations in a present day midsize tokamak devoted to a physics program still mainly oriented for basics of fusion. The system as described here has not yet arrived at its final goal, namely to deliver pellets at a size just sufficient to trigger ELM but avoiding fueling as much as possible. Thus, a pellet source foreseen to deliver even smaller pellets than the present one is still under construction. However, it turns out that reliable production, handling, and transfer of pellets through guiding tube systems become more and more troublesome when approaching the submillimeter-scale regime. As there are limitations set by the available conventional (and reliable) technology, the pacing approach in a tokamak experiment in the size range of ASDEX Upgrade will always be hampered by the fact that it is associated with significant particle flux potentially unfavorable for the plasma performance. Indeed, this caveat arises essentially due to the relatively small machine size! For bigger machines there will be a pivotal release of this problem due to two facts. First, the absolute pellet size gradually becomes ignorable with increasing plasma particle inventory of the target plasma. For example, a millimeter-sized pellet carries a particle amount on the order of several $10^{-2}$ the total plasma particle inventory in ASDEX Upgrade and can induce significant and clearly visible impacts on relevant plasma parameters. Already at JET, the same pellet causing only a particle increase by about $10^{-3}$ would be at the detection limit for global plasma parameters, while at ITER with a corresponding ratio of $10^{-4}$ it would be essentially ignorable. Fortunately, it already seems that pellets in the millimetersize range are sufficient to trigger ELMs even in machines as big as ITER, provided the imposed local perturbation is sufficiently strong. Experimental investigations at ASDEX Upgrade showed a rather shallow penetration about halfway to the plasma pedestal top is sufficient. ${ }^{25}$ This finding probably can be adopted for scaling up to ITER size. Therefore, it seems that available millimeter-sized pellets are oversized for ELM triggering by about a factor of 100 in a machine the size of ASDEX Upgrade, but would fit almost perfectly to a reactor-size plasma. Relaxing the refueling constraint also stems from reducing the intrinsic ELM frequency with growing machine size. For example, $f_{\mathrm{ELM}}^{0}$ is expected in the range $0.2-0.5 \mathrm{~Hz}$ for a standard type-I ELM operational scenario in ITER, ${ }^{9,26}$ while at ASDEX Upgrade we have to deal with $f_{\text {ELM }}$ in the range $20-200 \mathrm{~Hz}$. To reach sufficient ELM mitigation in ITER a pellet rate up to $4 \mathrm{~Hz}$ seems sufficient, ${ }^{26}$ while at ASDEX Upgrade pellets of at least $50 \mathrm{~Hz}$ are required for corresponding investigations. Altogether, the same particle flux or even a significantly lower one than applied in the experiments at ASDEX Upgrade is predicted to be adequate to achieve pellet pacing in the reactor grade ITER 
approach. At this plasma scale the respective fueling is no longer of any importance for the plasma performance. It therefore seems fair to state that pellet handling becomes easier for bigger machines, and pellet-based ELM pacing is a promising technique for future application in fusion research at a reactor grade level. Furthermore, the headroom introduced by growing machine size will allow combined goals of pellet injection, e.g., by simultaneous fueling and pacing choosing appropriate pellet parameters. ${ }^{26}$

${ }^{1}$ S. L. Milora et al., Nucl. Fusion 35, 657 (1995).

${ }^{2}$ L. Baylor et al., Nucl. Fusion 32, 2177 (1992).

${ }^{3}$ M. Kaufmann et al., Nucl. Fusion 26, 171 (1986).

${ }^{4}$ H. W. Müller et al., Phys. Rev. Lett. 83, 2199 (1999).

${ }^{5}$ P. T. Lang et al., Nucl. Fusion 36, 1531(1996); P. T. Lang et al., Nucl. Fusion 36, 153(E) (1996)

${ }^{6}$ P. T. Lang et al., Phys. Rev. Lett. 79, 1487 (1997).

${ }^{7}$ J. Pamela et al., Nucl. Fusion 45, S63 (2005).

${ }^{8}$ F. Federici et al., Plasma Phys. Controlled Fusion 45, 1523 (2003).

${ }^{9}$ A. Herrmann, Plasma Phys. Controlled Fusion 44, 897 (2002).
${ }^{10}$ P. T. Lang et al., Nucl. Fusion 43, 1110 (2003).

${ }^{11}$ L. D. Horton et al., 20th IAEA Conference, Vilamoura 2004, EX/P3-4.

${ }^{12}$ A. Kallenbach et al., J. Nucl. Mater. 337-339, 732 (2005).

${ }^{13}$ P. T. Lang et al., Rev. Sci. Instrum. 74, 3974 (2003).

${ }^{14}$ S. Combs, Rev. Sci. Instrum. 64, 1679 (1993).

${ }^{15}$ R. Gilliard et al., Rev. Sci. Instrum. 52, 183 (1981).

${ }^{16}$ A. Kallenbach et al., Nucl. Fusion 39, 901 (1999).

${ }^{17}$ R. Neu et al., Nucl. Fusion 45, 209 (2005).

${ }^{18}$ J. G. Leidenfrost, De Aquae Communis Nonnullis Qualitatibus Tractatus (Johann Straube, Duisburg, Germany, 1756). The Leidenfrost phenomenon is referred to as the levitation and sustainment of a cold body over a hot surface by establishing an insulating gas cushion. Originally observed and explained for levitation and sustainment of water drops on a hot plate by J.G. Leidenfrost, German scientist (1715-1794).

${ }^{19}$ V. Mertens et al., Fusion Sci. Technol. 44, 593 (2003).

${ }^{20}$ A. Herrmann and O. Gruber, Fusion Sci. Technol. 44, 569 (2003).

${ }^{21}$ ASDEX Upgrade Team, Fusion Sci. Technol. 44, 569 (2003).

${ }^{22}$ R. Neu, Phys. Scr. T123, 33 (2006).

${ }^{23}$ G. Kocsis et al., Rev. Sci. Instrum. 75, 4754 (2004).

${ }^{24}$ A. Lorenz et al., Fusion Eng. Des. 69, 15 (2003).

${ }^{25}$ P. T. Lang et al., Plasma Phys. Controlled Fusion 48, A141 (2006).

${ }^{26}$ A. Polevoi et al., Nucl. Fusion 43, 1072 (2003). 